\title{
Safety of GH after treatment for childhood cancer
}

\section{Hanneke M van Santen}

Pediatric Endocrinology, Princess Màxima Center for Pediatric Oncology and Wilhelmina Children's Hospital, Utrecht, the Netherlands

Correspondence should be addressed to $\mathrm{H}$ M van Santen Email

h.m.vansanten@ umcutrecht.nl
In an article in a recent issue of this journal, ThomasTeinturier et al. describe the influence of growth hormone (GH) therapy on the development of second neoplasms in childhood cancer survivors (CCS) (1). The manuscript is an important contribution to literature, as GH deficiency (GHD) is frequently observed in CCS, especially in the childhood brain tumor survivors after exposure to cranial irradiation or in children surviving a tumor in the hypothalamic-pituitary region. In their study, no increased risk was found for recurrence of the original tumor, nor for the development of a second tumor. A slight non-significant increased risk is described for the development of meningioma, which may be confounded by the previous radiotherapy. These results are reassuring for children who have been diagnosed with GHD after surviving a malignancy and who are treated with $\mathrm{GH}$ injections on a daily basis. There are, however, still some concerns and unanswered questions that need attention in future studies.

\section{General arguments of a relationship between GH treatment and cancer risk and the role of IGF1 in neoplasm development}

Safety of treatment with GH in CCS with GHD has been a debate for many years. GH increases IGF1 concentration, by which the both the positive metabolic effects and linear growth are affected. The intended rise in IGF1 concentration has however also raised concerns with regard to possible cancer risk. It has been reported that humans with isolated GHD type $1 \mathrm{~B}$, owing to a mutation of the GH-releasing hormone receptor gene, in the Itabaianinha County are, to a certain degree, protected from cancer (2). These individuals have low GH and IGF1 levels and are characterized by proportional short stature, doll facies, high-pitched voices, central obesity, have delayed puberty but are fertile and generally healthy. Studies performed in such populations are unique and enable the analysis of the impact of the severe reduction in GH signaling and subsequently low IGF1 levels on agerelated characteristics, health, and cancer risk. On the opposite, in individuals with prolonged increased IGF1 concentrations such as in acromegaly, an increased risk for cancer (thyroid, colon, breast) has been described (3). The exact role of GH and IGF1 on tumor development is still unclear and therefore there is still debate whether $\mathrm{GH}$ treatment in this vulnerable group patients will increase their risk to develop neoplasms.

\section{The risk of developing neoplasms during or after GH treatment in other conditions}

In children with short stature, without underlying endocrine conditions such as children born small for gestational age (SGA), idiopathic short stature or Turner syndrome, GH treatment has not been associated with increased cancer risk (4). Also, for non-CCS GHD children, no increased risk to develop cancer was reported $(4,5)$. The results of studies in these children cannot be compared to CCS, however, as CCS may have a different genetic profile or may have been treated with other toxic agents such as chemotherapy or radiotherapy, possibly influencing their (secondary) cancer risk. 


\section{Positive effects of GH treatment in CCS}

GH treatment in children diagnosed with GHD after surviving a malignancy may be indicated for several reasons. One of the main aims of GH treatment is to improve adult height; a health issue that may be considered less relevant when a child is fighting cancer, however becomes more important after finalizing cancer treatment, especially for the developing teenager or the young adolescent cancer survivor. To achieve an adult height comparable to sibs or peers may aid in self-confidence, self-image and help in the feelings of belonging to a peer group. Next to adult height, GH treatment may (partially) prevent or reverse negative metabolic effects of prolonged GHD, such as decreased muscle strength, lean body mass, lipid profile and bone health (6). To reverse these adverse metabolic consequences of GHD may be of special importance for CCS, due to the fact that many survivors may already be at risk for deprived metabolic outcome as a consequence of decreased mobility, periods of anorexia or cardiovascular toxicity. Treatment with GH may contribute to an overall better quality of life.

\section{Critical reflection on the methods used in the study by Thomas-Teinturier et $a$ l.}

The results of the study performed by Thomas-Teinturier et al. are reassuring (1), but need confirmation in welldesigned prospective studies as there are still multiple questions that need to be addressed concerning the treatment with GH in this specific population. To be able to answer these questions, it is important that detailed information is provided in reported cohorts and the same outcome measures are used across the different studies. This will enable meta-analysis of data.

One example of detailed information that was lacking in this report (and often is in other reports), is an exact description of patients. Of the studied cohort treated with GH, 14.3\% of patients had 'another type of tumor', but this is not further elaborated upon and also patients with 'benign' craniopharyngioma were included. It is uncertain whether the 'miscellaneous' tumors were benign or malignant. It is important to specify tumor types and not combine the outcomes of children treated for benign and malignant neoplasms. Subsequent cancer risk for patients surviving malignant disease maybe different than for patients surviving benign neoplasms, due to different genetic background or less exposure to cytotoxic agents.
Secondly, it is important to know the exact time span between the complete remission of the studied individuals and the start of GH treatment. In many studies that report on safety outcomes after GH treatment in CCS, a median range for starting $\mathrm{GH}$ treatment is provided. It is unclear, however, if the time span between achieving complete remission and start of $\mathrm{GH}$ treatment in the individual patient or that of the study cohort can be related to recurrence of the primary tumor or to the development of a secondary tumor. This is however, an issue of major importance that should be addressed in future studies. In most current recommendations, it has been suggested to delay treatment with GH until the patient has been 1 year disease-free following completion of therapy for malignant disease (7), although evidence to support this recommendation is low. It may be questioned whether 1 year is needed, or that waiting for 2 years is even better. The variables on which the time span between achieving complete remission and commencing $\mathrm{GH}$ treatment should be based on may be discussed. For the child with IGF1 concentrations $<-2$ SD in combination with obvious metabolic signs of GHD (high BMI, high pitched voice, growth arrest), it may be more beneficial to start $\mathrm{GH}$ treatment more early, such as 3-6 months after achieving complete remission. This may also be the case for a child entering puberty in whom a year without $\mathrm{GH}$ has direct important negative consequences for adult height. In the study by Thomas-Teinturier and colleagues, the period for the start of $\mathrm{GH}$ treatment was provided (from -2 to 22.5 years) which raises several questions. It is not clearly described if the patients who had been treated with $\mathrm{GH}$ before cancer diagnosis, stopped their GH treatment or when it was restarted. Also the average time of starting $\mathrm{GH}$ after reaching complete remission is not clear. It is important that detailed clinical and biochemical information is provided to enable meta-analysis of data.

An even more difficult question is the one concerning GHD in children with stable disease or frequently recurring disease, such as children with low-grade glioma. There are insufficient studies to make an evidence-based statement upon GH treatment in this specific population. Studies in these populations are extremely challenging due to the fact that children with slowly progressive lowgrade glioma often receive multiple chronic and changing regimens of chemotherapy. Yet, these questions do arise in the neuro-oncology-multi-disciplinary clinic and need to be answered for patients, parents and treating physicians dealing with the child in growth arrest due to GHD, sometimes in combination with poor metabolic 
state due to hypothalamic dysfunction in combination with low-grade oncologic disease. To provide us with such answers and harmonize treatment with GH therapy across the world, the international guideline harmonization group (IGHG (8)) together with a large group of experts, has recently started a working group aiming to form a joint recommendation upon GH treatment in children surviving cancer.

A third important outcome that might have improved this study if it were included, is the concentration of IGF1 during GH treatment. This information is needed to analyze the association between the concentration of IGF1 and the risk to develop a subsequent malignancy. This has hardly been studied. Although the results of Thomas-Teinturier et al. are reassuring, showing no increased cancer risk, it could be hypothesized that restoring the IGF1 to 0 SD has a different outcome than increasing it to $+2 \mathrm{SD}$ or even higher. Prospective or even retrospective analyses of large cohorts including the analysis of longitudinal, repeated IGF1 concentrations in relation to subsequent cancer or recurrence of disease may provide an answer.

\section{Comparing the results with other published studies}

Several other studies reporting on tumor recurrence or secondary tumors have been published, mostly in agreement with the result by Thomas-Teinturier et al., although there also have been some reports of increased risk for certain cancer types or with a trend toward increased risk of mortality $(4,5,9,10)$. The studies reporting an increased risk of secondary cancer or mortality, must be interpreted precociously for three reasons; first, in most studies, due to the relatively small number of events, high confidence intervals and subsequent low levels of significance are present; secondly in many studies, the used control groups resulted in a high risk of outcome bias due to the fact that patients surviving childhood cancer receiving GH treatment already were at increased risk for mortality or a secondary neoplasms due to their history with more toxic therapies such as high dose chemotherapy, stem cell transplantation or radiotherapy. The increased cause-specific mortality of the children with cancer reported in the recent SAGhe study (4), was also interpreted to be associated with the underlying diagnosis. Favorably, future studies should be performed in a homogenous cancer survivor group, with the same tumor type and toxicity all with GHD, with and without GH treatment. Unfortunately, such studies are not available. Thirdly, regarding the reported trend toward increased mortality, no significant relation of GH-dose or duration of GHRT on mortality was found, making a causal relation less likely $(4,5)$. Nevertheless, GH therapy in high-risk patients should be given with care and the decision to start GH therapy should be carefully discussed with families (9).

\section{Conclusions}

In conclusion, when combining the results of the study by Thomas-Teinturier et al. with previous reports, the conclusion may be drawn that CCS treated with GH seem not to be at increased risk for recurrence of the original tumor, nor for a second tumor or meningioma compared to CCS not treated with GH. Future prospective studies are needed though to confirm these results and to answer the more difficult question upon the optimal time span to start GH therapy after achieving complete remission and how to deal with children with 'chronic' low-grade oncologic disease and GHD. Also, more studies are needed upon the optimal dosing of GH treatment.

Declaration of interest

The author declares that there is no conflict of interest that could be perceived as prejudicing the impartiality of this commentary.

\section{Funding}

This research did not receive any specific grant from any funding agency in the public, commercial or not-for-profit sector.

\section{References}

1 Thomas-Teinturier C, Oliver-Petit I, Pacquement H, Fresneau B, Sétchéou Allodji RS, Veres C, Bolle S, Berchery D, DemoorGoldschmidt C, Haddy N et al. Influence of growth hormone therapy on the occurrence of a second neoplasm in survivors of childhood cancer. European Journal of Endocrinology 2020183 471-480. (https:// doi.org/10.1530/EJE-20-0369)

2 Aguiar-Oliveira MH \& Bartke A. Growth hormone deficiency: health and longevity. Endocrine Reviews 201940 575-601. (https://doi. org/10.1210/er.2018-00216)

3 Wolinski K, Stangierski A, Dyrda K, Nowicka K, Pelka M, Iqbal A, Car A, Lazizi M, Bednarek N, Czarnywojtek A et al. Risk of malignant neoplasms in acromegaly: a case-control study. Journal of Endocrinological Investigation 201740 319-322. (https://doi. org/10.1007/s40618-016-0565-y)

4 Sävendahl L, Cooke R, Tidblad A, Beckers D, Butler G, Cianfarani S, Clayton P, Coste J, Hokken-Koelega ACS, Kiess W et al. Long-term mortality after childhood growth hormone treatment: the SAGhE 
cohort study. Lancet: Diabetes and Endocrinology 20208 683-692. (https://doi.org/10.1016/S2213-8587(20)30163-7)

5 Swerdlow AJ, Cooke R, Beckers D, Borgström B, Butler G, Carel JC, Cianfarani S, Clayton P, Coste J, Deodati A et al. Cancer risks in patients treated with growth hormone in childhood: the SAGhE European cohort study. Journal of Clinical Endocrinology and Metabolism 2017102 1661-1672. (https://doi.org/10.1210/jc.2016-2046)

6 Bang P. Pediatric implications of normal insulin-GH-IGF-axis physiology. In Eds KR Feingold, B Anawalt, A Boyce, G Chrousos, WW de herder, K Dungan, A Grossman, JM Hershman, HJ Hofland, G Kaltsas, C Koch, P Kopp, M Korbonits, R McLachlan, J E Morley, M New, J Purnell, F Singer, C A Stratakis, DL Trence and D P Wison www.endoctext.com. 2000.

7 Sklar CA, Antal Z, Chemaitilly W, Cohen LE, Follin C, Meacham LR $\&$ Murad MH. Hypothalamic-pituitary and growth disorders in survivors of childhood cancer: an Endocrine Society clinical practice guideline. Journal of Clinical Endocrinology and Metabolism 2018103 2761-2784. (https://doi.org/10.1210/ jc.2018-01175)
8 Kremer LC, Mulder RL, Oeffinger KC, Bhatia S, Landier W, Levitt G, Constine LS, Wallace WH, Caron HN, Armenian SH et al. A worldwide collaboration to harmonize guidelines for the long-term follow-up of childhood and young adult cancer survivors: a report from the International Late Effects of Childhood Cancer Guideline Harmonization Group. Pediatric Blood and Cancer 201360 543-549. (https://doi.org/10.1002/pbc.24445)

9 Allen DB, Backeljauw P, Bidlingmaier M, Biller BMK, Boguszewski M, Burman P, Butler G, Chihara K, Christiansen J, Cianfarani S et al. GH safety workshop position paper: a critical appraisal of recombinant human GH therapy in children and adults. European Journal of Endocrinology 2016174 P1-P9. (https:// doi.org/10.1530/EJE-15-0873)

10 Sklar CA, Mertens AC, Mitby P, Occhiogrosso G, Qin J, Heller G, Yasui Y \& Robison LL. Risk of disease recurrence and second neoplasms in survivors of childhood cancer treated with growth hormone: a report from the Childhood Cancer Survivor Study. Journal of Clinical Endocrinology and Metabolism 200287 3136-3141. (https://doi.org/10.1210/jcem.87.7.8606)

Received 24 August 2020

Received in final form 24 September 2020

Accepted 30 September 2020 